\title{
EphA2 silencing in nasopharyngeal carcinoma leads to decreased proliferation, invasion and increased sensitization to paclitaxel
}

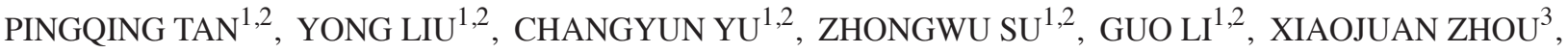 \\ DONGHAI HUANG ${ }^{1,2}$, XIN ZHANG $^{1,2}$, YUANZHENG QIU ${ }^{1,2}$ and YONGQUAN TIAN ${ }^{1,2}$ \\ ${ }^{1}$ Department of Otolaryngology, Head and Neck Surgery, Xiangya Hospital, Central South University; \\ ${ }^{2}$ Otolaryngology Major Disease Research Key Laboratory of Hunan Province, Changsha, Hunan 410008; \\ ${ }^{3}$ Department of Otolaryngology, Changsha No.1 Hospital, Changsha, Hunan 410013, P.R. China
}

Received January 31, 2012; Accepted May 22, 2012

DOI: $10.3892 / 01.2012 .746$

\begin{abstract}
EphA2 is frequently overexpressed and functionally altered in a variety of human cancers. However, its roles in human nasopharyngeal carcinoma (NPC) remain unclear. To investigate the roles of EphA2 in the development and progression of NPC, we initially evaluated the expression pattern of EphA2 protein in NPC tissues using western blotting and CCK-8 assay. Fluorescence-activated cell sorting analysis and invasion assay were conducted to observe the effects of EphA2 inhibition in vivo. Our results demonstrated that EphA2 was overexpressed in NPC specimens and the expression of EphA2 was significantly associated with T classification, advanced clinical stage and lymph node metastasis. Moreover, human NPC 5-8F cells were infected with lentiviral vector-mediated EphA2-specific shRNA, which resulted in the significant inhibition of cell growth, invasion of 5-8F cells and markedly enhanced the sensitivity of 5-8F cells to the chemotherapeutic agent paclitaxel in vitro. Collectively, our results demonstrate that EphA2 is involved in malignant cell behavior and is a potential therapeutic target in human NPC.
\end{abstract}

\section{Introduction}

Nasopharyngeal carcinoma (NPC), an Epstein-Barr virus (EBV)-associated cancer, is highly prevalent in southeast Asia, especially in the Cantonese region around Guangzhou in China (1). NPC is distinct from other cancers of the head and neck in terms of its epidemiology, histopathology, clinical characteristics, methods of treatment and patterns of failure (2). Although patients benefit from multi-modal treatment,

Correspondence to: Professor Yongquan Tian or Dr Yuanzheng Qiu, Department of Otolaryngology, Xiangya Hospital, Central South University, Xiangya Road 87, Changsha, Hunan 410008, P.R. China

E-mail: 414570540@qq.com; braver19840112@foxmail.com

Key words: nasopharyngeal carcinoma, EphA2, metastasis, drug resistance, paclitaxel including radiotherapy, chemotherapy and biological therapy, limited improvement in 5-year survival has been achieved over the last few decades (3). Cancer metastasis and therapy failure account for the low survival rates in patients with NPC $(4,5)$. However, the molecular mechanism of the development and progression of NPC remains poorly understood. It is of great clinical value to understand the molecular mechanism of this cancer and identify valuable predictive markers as well as novel therapeutic strategies.

The Eph protein family constitutes the largest group of transmembrane receptor tyrosine kinases (RTKs) identified in the genome. Depending on sequence homology and binding affinity of the two different types of membrane-anchored ephrin ligands, the Eph family may be classified as EphA or EphB. Eph kinases have been extensively studied for their roles in embryonic development where they transduce key directional signals for cell positioning, axon guidance, tissue border formation, vascular development and cancer $(6,7)$.

EphA2, a member of the Eph kinase family, originally designated as epithelial cell kinase (Eck), is usually expressed at low levels in adult epithelial tissues (8). Mounting evidence suggests EphA2 overexpression in cell transformation, primary tumor initiation, progression, angiogenesis and metastasis in a variety of cancer models $(9,10)$. In non-transformed mammary epithelial cells, the ectopic overexpression of EphA2 has been shown to result in a malignant phenotype in in vitro and in vivo contexts (11). Cancer cells that overexpress EphA2 exhibit increased motility and invasive properties, consistent with a pro-metastatic phenotype (12). EphA2 overexpression is prevalent in numerous solid tumors, including ovarian $(13,14)$, lung $(15)$, glioblastoma (16), prostate (17), and renal tumors (18). Moreover, a high EphA2 expression is correlated with disease stage, increased tumor metastasis, and poor patient survival, suggesting EphA2 to be a candidate prognostic marker for a variety of human malignancies. However, few published reports have evaluated the role of EphA2 expression in NPC, especially its effects on the malignant behavior of NPC in vitro.

To gain better insight into the clinical relevance of EphA2 in NPC, the present study was carried out to investigate EphA2 expression patterns in NPC tissue samples and assess whether EphA2 expression is correlated with clinico- 
Table I. Correlation between the clinicopathological characteristics and protein expression of EphA2.

\begin{tabular}{|c|c|c|c|c|}
\hline Characteristics & No. of samples & Relative protein expression of EphA2 & t-value & P-value \\
\hline \multicolumn{5}{|l|}{ Age (y) } \\
\hline$<46$ & 24 & $0.86 \pm 0.37$ & \multirow[t]{2}{*}{0.959} & \multirow[t]{2}{*}{0.343} \\
\hline$\geq 46$ & 23 & $0.76 \pm 0.30$ & & \\
\hline \multicolumn{5}{|l|}{ Gender } \\
\hline Male & 37 & $0.79 \pm 0.33$ & \multirow[t]{2}{*}{0.571} & \multirow[t]{2}{*}{0.571} \\
\hline Female & 10 & $0.86 \pm 0.36$ & & \\
\hline \multicolumn{5}{|l|}{ Smoking history } \\
\hline Smoker & 29 & $0.86 \pm 0.35$ & \multirow[t]{2}{*}{1.423} & \multirow[t]{2}{*}{0.162} \\
\hline Nonsmoker & 18 & $0.72 \pm 0.29$ & & \\
\hline \multicolumn{5}{|l|}{$\mathrm{T}$ classification } \\
\hline $\mathrm{T} 1+\mathrm{T} 2$ & 20 & $0.68 \pm 0.28$ & \multirow[t]{2}{*}{2.272} & \multirow[t]{2}{*}{0.028} \\
\hline $\mathrm{T} 3+\mathrm{T} 4$ & 27 & $0.90 \pm 0.34$ & & \\
\hline \multicolumn{5}{|l|}{ Clinical stage } \\
\hline $\mathrm{I}+\mathrm{II}$ & 19 & $0.64 \pm 0.21$ & \multirow[t]{2}{*}{3.069} & \multirow[t]{2}{*}{0.004} \\
\hline $\mathrm{III}+\mathrm{IV}$ & 28 & $0.92 \pm 0.36$ & & \\
\hline \multicolumn{5}{|l|}{ Metastasis } \\
\hline Yes & 27 & $0.91 \pm 0.34$ & \multirow[t]{2}{*}{2.616} & \multirow[t]{2}{*}{0.012} \\
\hline No & 20 & $0.67 \pm 0.28$ & & \\
\hline \multicolumn{5}{|c|}{ EB-virus infection } \\
\hline Yes & 26 & $0.87 \pm 0.34$ & \multirow[t]{2}{*}{1.338} & \multirow[t]{2}{*}{0.188} \\
\hline No & 21 & $0.74 \pm 0.32$ & & \\
\hline
\end{tabular}

pathological parameters in patients with NPC. A lentiviral RNAi system was employed to knock down the expression of EphA2 in NPC 5-8F cells. The roles of EphA2 in NPC cell invasion and chemotherapy in vitro conditions were investigated.

\section{Materials and methods}

Patients and tissue preparation. A total of 47 fresh undifferentiated NPC (WHO type III) tissues and 21 cases of non-carcinoma epithelial tissues (NCET) from the nasopharynx were obtained from the Department of Otolaryngology of Xiangya Hospital, Central South University, China, between October 2010 and March 2011. None of the patients had any history of previous malignancies, or history of radiotherapy or chemotherapy. Metastases were diagnosed by clinical examination and imaging evaluation. The clinical stage of all the patients was classified according to the 2008 NPC staging system of China. Specimens were snap-frozen immediately and stored in liquid nitrogen for total protein extraction. The study was approved by the Research Ethics Committee of Central South University, Changsha, China. Informed consent was obtained from all patients. The specimens were handled and anonymized according to ethical and legal standards. Clinical information for all the NPC samples is described in Table I.

Western blotting. The western blot analyses were performed as described in previous studies $(19,20)$. In brief, total protein $(50 \mu \mathrm{g} / \mathrm{sample})$ was extracted and separated by $12 \%$ sodium dodecyl sulfate-polyacrylamide gel electrophoresis
(SDS-PAGE) and then transferred onto PVDF membranes (Millipore, Bedford, MA, USA). The blotted membranes were incubated with rabbit poly-antibody against EphA2 (sc-924, dilution 1:400, Santa Cruz Biotechnology, Santa Cruz, CA, USA) and then secondary antibody (Beyotime, China), respectively. $\beta$-actin protein was also determined by using specific antibody (Beyotime) as a loading control.

Cell culture and reagents. NPC 5-8F cells were maintained as monolayer cultures in RPMI-1640 supplemented with $10 \%$ fetal bovine serum (FBS), $100 \mathrm{IU} / \mathrm{ml}$ penicillin and $100 \mathrm{IU} / \mathrm{ml}$ streptomycin at $37^{\circ} \mathrm{C}$ in a humidified atmosphere with $5 \%$ $\mathrm{CO}_{2}$. Exponentially growing cells were used for the subsequent experiments.

Lentiviral vectors mediated EphA2-specific shRNA transfection. EphA2-specific shRNA lentiviral particles (sc-29304-V, Santa Cruz) are pools of concentrated, transduction-ready viral particles containing three target-specific constructs that encode 19-25 nt (plus hairpin) shRNA designed to knock down EphA2 gene expression in human NPC 5-8F cells. 5-8F cells $\left(2 \times 10^{4}\right)$ were seeded into 24 -well plates and allowed to grow at $80 \%$ confluence. Medium containing EphA2 or control shRNA lentiviral particles was added to these cells. Twelve hours later, the original medium was replaced with fresh complete medium and the cells were subjected to silencing efficiency assay via western blotting $72 \mathrm{~h}$ post-infection.

Cell proliferation assay. A Cell Counting Kit-8 (Beyotime) was employed to draw cell growth curves according to the 
manufacturer's instructions. In brief, cells at a concentration of $3.0 \times 10^{3} /$ well were cultured in triplicate in 96 -well plates with $10 \% \mathrm{FBS}$ at $37^{\circ} \mathrm{C}$ in humidifed $5 \% \mathrm{CO}_{2}$ atmosphere for various periods and exposed to fresh media every other day. At 24, 48, 72 and $96 \mathrm{~h}$ post-transfection, cell proliferation was measured by absorbance at an optical density of $490 \mathrm{~nm}$.

Cell apoptosis and cell cycle analysis by flow cytometry. Fluorescence-activated cell sorting analysis was carried out. Following incubation in serum-free medium for $24 \mathrm{~h}$, the cells were washed with PBS at $4^{\circ} \mathrm{C}$ twice and their concentration was altered to $1 \times 10^{6} / \mathrm{ml}$. Annexin V/Cy5 and propidium iodide were added for incubation for $30 \mathrm{~min}$. Fluorescence-activated cell sorting analysis was carried out using the FACS Calibur instrument (Becton Dickinson, Franklin Lakes, NJ, USA).

Matrigel invasion assay. The invasiveness of the transfected cells was evaluated in 24-well transfected chambers (Costar, Cambridge, MA, USA) according to the manufacturer's instructions. Briefly, transwell with an $8 \mu \mathrm{m}$ diameter pore membrane was coated with $200 \mu \mathrm{l}$ matrigel at $200 \mu \mathrm{g} / \mathrm{ml}$ and incubated overnight. Cells $\left(3 \times 10^{4}\right)$ in $100 \mu$ l of serum-free medium were seeded into the upper chamber of the transwell and the lower chamber was filled with $0.8 \mathrm{ml}$ RPMI-1640 containing $12 \%$ FBS to induce chemotaxis. Following $48 \mathrm{~h}$ of incubation at $37^{\circ} \mathrm{C}$ in a humidifed $5 \% \mathrm{CO}_{2}$ atmosphere, the cells were fixed in methanol and stained with crystal violet, and the cells that invaded through the pores to the lower surface of the filter were counted under a microscope. Three invasion chambers were used per condition. The values obtained were calculated by averaging the total number of cells from three filters.

Cell viability assay. Cells were seeded in 96-well plates. Following overnight culture, they were exposed to various concentrations of paclitaxel $(0.001,0.01,0.1,1.5,10,20$ and $30 \mathrm{nM}$ ) for $48 \mathrm{~h}$ in a $\mathrm{CO}_{2}$ incubator. CCK8 assay was used to detect the chemo-sensitivity of cells. Absorbance values were expressed as percentages relative to the controls, and the concentrations resulting in 50\% inhibition of cell growth ( $\mathrm{IC}_{50}$ values) were calculated.

Statistical analysis. Statistical analysis was performed with SPSS 17.0 software. Results of quantitative data in this study were shown as the mean \pm SD. Statistical differences between groups were compared using the two-tailed Student's t-test. $\mathrm{P}<0.05$ was considered statistically significant.

\section{Results}

Overexpression of EphA2 protein in NPC and its correlation with clinicopathological parameters. The expression pattern of EphA2 protein was initially assayed in 47 fresh NPC specimens and 21 non-carcinoma epithelial tissue (NCET) specimens. Western blot analysis clearly revealed that the expression of EphA2 protein in NPC tissues was significantly higher than that in NCET $(0.81 \pm 0.33$ vs. $0.43 \pm 0.21 ; \mathrm{t}=4.841$, $\mathrm{P}<0.001$ ) (Fig. 1). Furthermore, the association between the expression of EphA2 protein and clinicopathological characteristics of NPC was explored using the Student's t-test. EphA2 overexpression was significantly associated with NPC
A

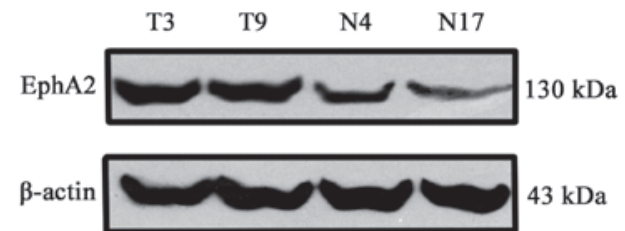

B

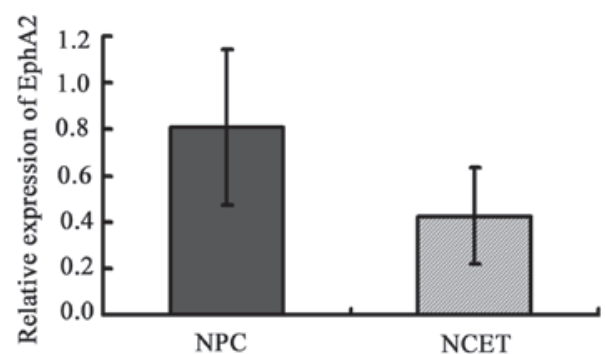

Figure 1. Protein expression of EphA2 in nasopharyngeal carcinoma (NPC) and non-carcinoma epithelial tissues (NCET, used as a control) from the nasopharynx. The protein abundance of EphA2 relative to the levels of $\beta$-actin protein, respectively, is shown. T, tumor sample; N, non-carcinoma epithelia. (A) Representative western blot analysis results. (B) Relative protein expression levels of EphA2 in tissue specimens ( $0.81 \pm 0.33$ vs. $0.43 \pm 0.21)$ $(\mathrm{t}=4.841, \mathrm{P}<0.001)$

A

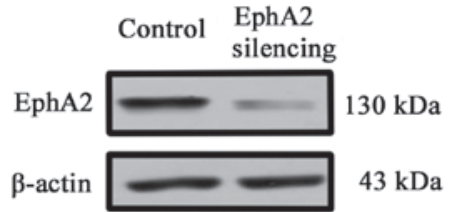

$\mathbf{B}$

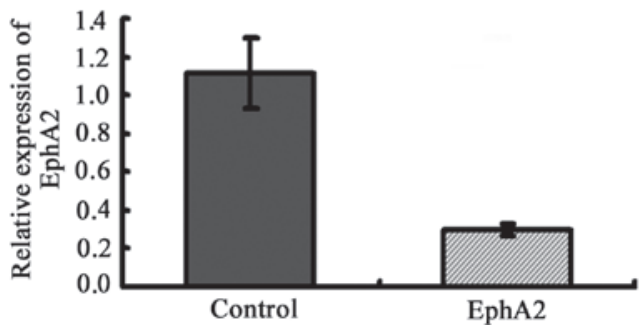

Figure 2. Knockdown of EphA2 expression by lentiviral vector-mediated shRNA in NPC 5-8F cells. (A) Downregulated protein expression of EphA2 detected by western blotting. (B) Graphical representation of the relative protein expression of EphA2 in the two groups of cells transfected with EphA2-shRNA and control-shRNA.

T classification $(\mathrm{P}=0.028)$, advanced clinical stages $(\mathrm{P}=0.004)$ and lymph node metastasis $(\mathrm{P}=0.012)$, respectively (Table I). However, no significant relationship existed between protein level of EphA2 and variables such as age $(\mathrm{P}=0.343)$, gender $(\mathrm{P}=0.571)$, smoking history $(\mathrm{P}=0.162)$ and the status of EB-virus infection $(\mathrm{P}=0.188)$.

EphA2 knockdown by EphA2 shRNA lentiviral particles in NPC 5-8F cells. To clarify the correlation of the expression of EphA2 protein and the biological process of NPC, we employed the lentivirus-delivered EphA2 or control shRNA to inhibit the expression of EphA2 in NPC 5-8F cells. Western blotting was carried out to assess the inhibition efficiency of EphA2 protein at $72 \mathrm{~h}$ following infection. Lentivirus- 

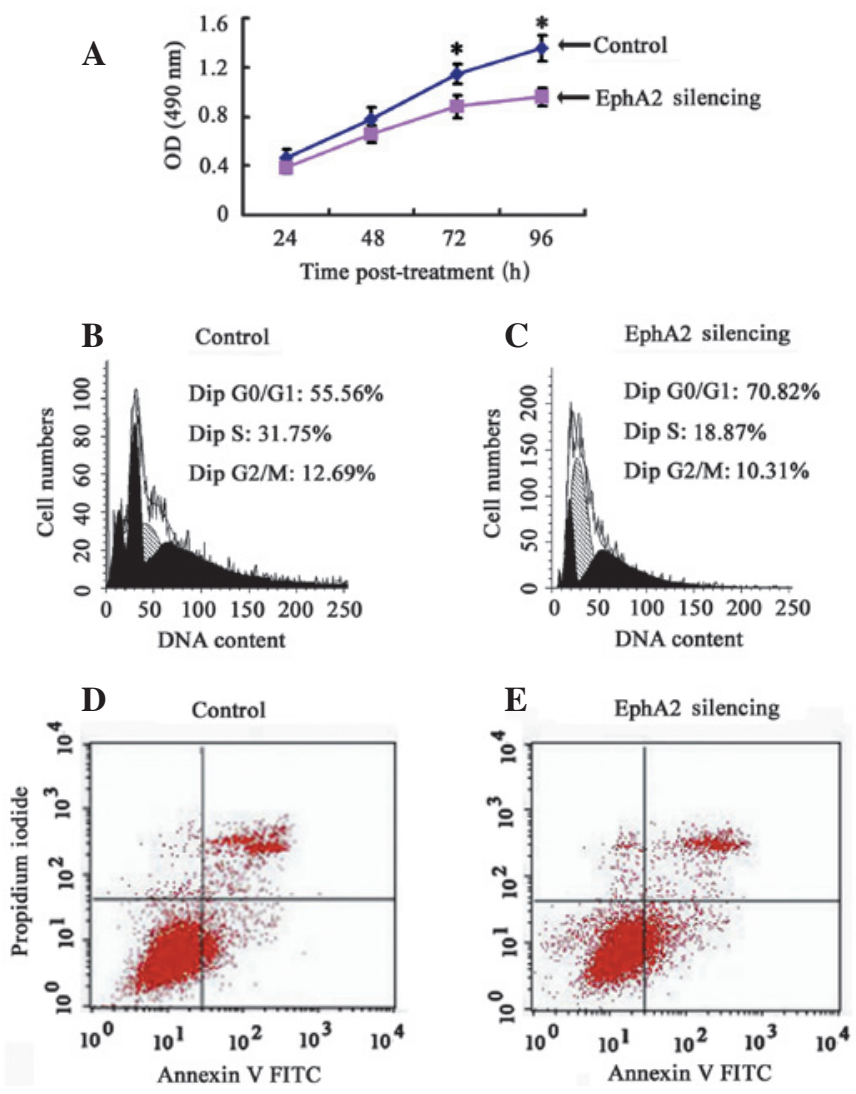

Figure 3. The effect of EphA2 on the proliferation, cell cycle distribution and apoptosis of NPC 5-8F cells. (A) Cell proliferation was measured by the CCK- 8 assay every $24 \mathrm{~h}$ for 4 days. (B and C) Differently treated cells were stained with propidium iodide and analyzed by flow cytometry. Proportion of cells in various phases of the cell cycle. The results are the means of three independent experiments $\pm \mathrm{SD}(\mathrm{P}<0.05)$. ( $\mathrm{D}$ and $\mathrm{E})$ Cells staining positive for Annexin V-FITC and negative for PI were considered to have undergone apoptosis. Average apoptotic rate of three independent experiments $\pm \mathrm{SD}$ are shown. ${ }^{*} \mathrm{P}<0.05$.

A

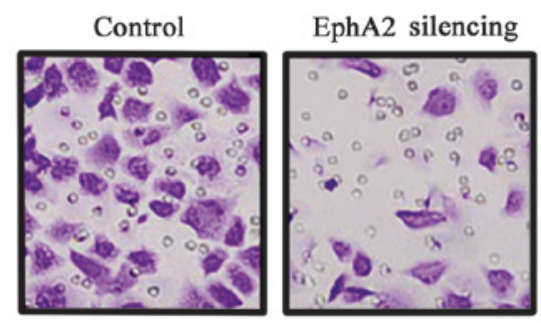

B

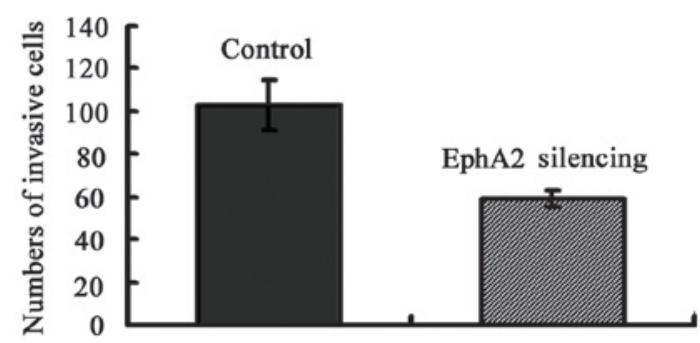

Figure 4. Knockdown of EphA2 inhibited the invasion of NPC 5-8F cells in vitro. (A) Transwell invasion assay showed that the knockdown of EphA2 inhibited cell invasion of 5-8F. Images were captured at a magnification of $x 200$. Representative of three independent experiments. (B) Graphical representation of the number of invasive $5-8 \mathrm{~F}$ cells per microscopic field. Data were shown as the mean \pm SD from three independent experiments.

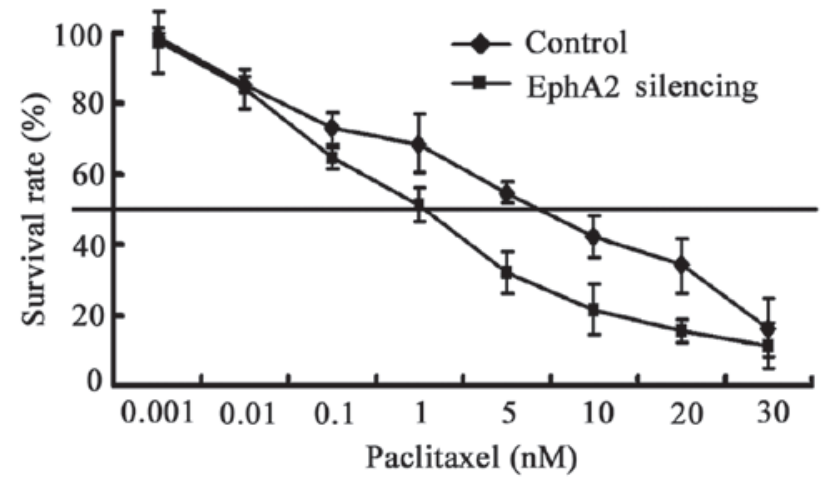

Figure.5 Knockdown the expression of EphA2 sensitized 5-8F cells to paclitaxel. The $\mathrm{IC}_{50}$ value of cells transfected with EphA2-shRNA and control-shRNA were 1.6 and $5.6 \mathrm{nM}$, respectively. Each experiment was performed in triplicate.

delivered EphA2 shRNA successfully resulted in $>70 \%$ inhibition efficiency in protein expression (Fig. 2).

EphA2 knockdown inhibits the proliferation of NPC 5-8F cells by inducing cell cycle arrest in the G0/G1 phase in vitro. To clarify the effect of EphA2 on the proliferation of NPC cells in vitro, a CCK-8 assay was performed and cell growth curve was generated. EphA2 knockdown inhibited the proliferation of NPC 5-8F cells in vitro, indicating that the expression of EphA2 affects the growth of NPC cells (Fig. 3A). To elucidate the mechanism of EphA2-mediated cell growth inhibition in NPC cells, flow cytometry was carried out to monitor cell cycle and apoptotic changes. The results demonstrated that, when compared with the control group, the percentage of NPC cells in the EphA2 silencing group in the G0/G1 phase was significantly increased (EphA2 silencing group: $74.38 \pm 3.71$ vs. the Control group: 53.81 \pm 2.19$)(\mathrm{P}<0.05)$, whereas the percentage of cells in the S phase (EphA2 silencing group: $17.89 \pm 2.21$ vs. the Control group: $30.41 \pm 1.55$ ) and G2/M phase (EphA2 silencing group: $7.72 \pm 2.24$ vs. the Control group: $15.80 \pm 3.72$ ) were obviously decreased $(\mathrm{P}<0.05)$ (Fig. 3B and $\mathrm{C})$. However, there was no significant difference in apoptotic rate (EphA2 silencing group: $7.01 \pm 1.06$ vs. the Control group: $5.78 \pm 1.14$, $)(\mathrm{P}>0.05)$ (Fig. 3D and E). These results demonstrate that knockdown of EphA2 induces G0/G1 phase arrest in NPC 5-8F cells, resulting in the growth inhibitory properties of NPC cells.

EphA2 knockdown inhibits the invasion of NPC 5-8F cells in vitro. To evaluate the function of EphA2 on NPC cell invasion, Matrigel invasion chambers were utilized. Inhibited EphA2 expression led to a significantly decreased invasive ability of NPC $5-8 \mathrm{~F}$ cells (EphA2 silencing group: $59 \pm 4$ vs. the Control group $103 \pm 12, \mathrm{P}=0.004$ ) (Fig. 4). These results clearly demonstrate that EphA2 are crucial in mediating cell invasion in NPC, which is a key determinant of NPC malignant progression and metastasis.

EphA2 knockdown sensitizes NPC 5-8F cells to paclitaxel in vitro. Chemotherapy is vital for the treatment of NPC, particularly neoadjuvant chemotherapy (21). In this study, we tested whether the downregulation of EphA2 was capable of sensitizing the NPC cell line 5-8F to paclitaxel, a widely 
used chemotherapeutic agent in NPC. 5-8F cells were exposed to paclitaxel following transfection with EphA2-shRNA for $48 \mathrm{~h}$. Cell viability was evaluated using CCK-8 assays. The $\mathrm{IC}_{50}$ value of cells transfected with EphA2-shRNA and controlshRNA were 1.6 and $5.6 \mathrm{nM}$, respectively (Fig. 5). The results show that the sensitivity of 5-8F to paclitaxel is enhanced by inhibiting the expression of EphA2 3.5-fold.

\section{Discussion}

Our previous investigations have clearly demonstrated EphA2 overexpression in human laryngeal and hypopharyngeal squamous cell carcinoma, and EphA2 regulation of the growth and cervical lymph node metastasis of human laryngeal and hypopharyngeal squamous cell carcinoma in vitro and in vivo $(20,22)$. Although NPC is also classified as a subtype of head and neck squamous cell carcinoma, it possesses unique and distinct epidemiology, clinical characteristics, etiology and histopathology (2). Therefore, the association between EphA2 expression and clinicopathological parameters in patients with NPC, and its role in growth, invasion and paclitaxel chemotherapy of NPC were investigated for the first time in the present study.

Results of the present study initially revealed that the expression level of EphA2 protein was significantly higher in NPC samples than that in non-carcinoma tissues obtained from the nasopharynx. Morever, the results of 47 NPC specimens assayed by western blotting further demonstrated that a high protein expression of EphA2 was significantly associated with T classification, clinical stage and cervical lymph nodes metastasis, which was consistent with recent publications on NPC (23) and other reports in urinary bladder cancer (24), ovarian cancer (13) and lung cancer $(25,26)$, indicating the importance of EphA2 in the malignant progression of patients with NPC.

Metastasis at the early stages is one of the most frequent clinical features in patients with NPC. Approximately 70-80\% of new patients with NPC present with cervical lymph node metastasis, and approximately $4.2 \%$ of those patients have distant metastasis to the bone, lung, liver, and central nervous system (27). Metastasis severely reduces the possibility of successful treatment and overall survival time (28). Therefore, identification of molecular markers for metastasis would be beneficial in designing optimized and individualized therapeutic regimens for patients with NPC. The present study revealed the association of EphA2 with lymph node metastasis in NPC, suggesting that EphA2 could be considered as an indicator for the propensity to metastasize. Furthermore, EphA2 inhibition was able to reduce the invasive ability of NPC 5-8F cells in vitro, supporting the theory that EphA2 is involved in the process of invasion and metastasis in patients with NPC.

Since acquired resistance to chemotherapy negatively affects the outcome of patients with NPC (29) and the finding that EphA2 knockdown led to inhibition of NPC proliferation in vitro, the potential therapeutic function of EphA2 in combination with a chemotherapeutic drug was further explored in the current study. Paclitaxel is a tubulin-stabilizing anti-cancer agent and now widely used in the therapy of patients with NPC. We found that knockdown of the EphA2 protein significantly enhanced the cytotoxicity of paclitaxel in human NPC
5-8F cells, which is a novel finding of the present study. Our results reveal that paclitaxel chemotherapy may be much more efficacious in human NPC when administered in combination with EphA2 gene inhibition. This result was consistent with a recent publication on ovarian cancer, in which EphA2 knockdown greatly improved the anticancer efficacy of paclitaxel in ovarian cancer in vitro and in vivo (30). However, the combined effect with paclitaxel chemotherapy needs to be further investigated in vivo.

In conclusion, the present study reveals that the EphA2 protein is overexpressed in NPC specimens and is associated with the clinical progression of patients with NPC. Moreover, the knockdown of EphA2 may significantly inhibit cell growth, invasion, and enhance the sensitivity to paclitaxel in vitro, indicating that EphA2 may be a valuable therapeutic target for patients with NPC. However, a deeper understanding of the precise mechanisms by which EphA2 regulates the above-mentioned processes is necessary.

\section{Acknowledgements}

This study was supported by grants from the National Natural Science Foundation of China (81172558, 81071757, 30872852 and 30901664), the Key Program of Natural Science Foundation of Hunan Province (2010TP4012-1), the Research Fund for the Doctoral Program of Higher Education of China (20100162110036, 20090162110065) and the Freedom Explore Program of Central South University (No. 2012QNZT099).

\section{References}

1. Fahraeus R, Fu HL, Ernberg I, et al: Expression of Epstein-Barr virus-encoded proteins in nasopharyngeal carcinoma. Int $\mathrm{J}$ Cancer 42: 329-338, 1988.

2. Shanmugaratnam K: Nasopharyngeal carcinoma: epidemiology, histopathology and aetiology. Ann Acad Med Singapore 9: 289-295, 1980 .

3. Jemal A, Siegel R, Xu J and Ward E: Cancer statistics. CA Cancer J Clin 60: 277-300, 2010.

4. Teo P, Shiu W, Leung SF and Lee WY: Prognostic factors in nasopharyngeal carcinoma investigated by computer tomography - an analysis of 659 patients. Radiother Oncol 23: 79-93, 1992 .

5. Tan EH, Khoo KS, Wee J, et al: Phase II trial of a paclitaxel and carboplatin combination in Asian patients with metastatic nasopharyngeal carcinoma. Ann Oncol 10: 235-237, 1999.

6. Pasquale EB: Eph receptors and ephrins in cancer: bidirectional signalling and beyond. Nat Rev Cancer 10: 165-180, 2010.

7. Pasquale EB: Eph-ephrin bidirectional signaling in physiology and disease. Cell 133: 38-52, 2008

8. Sulman EP, Tang XX, Allen C, et al: ECK, a human EPH-related gene, maps to $1 \mathrm{p} 36.1$, a common region of alteration in human cancers. Genomics 40: 371-374, 1997.

9. Brantley-Sieders DM, Zhuang G, Hicks D, et al: The receptor tyrosine kinase EphA2 promotes mammary adenocarcinoma tumorigenesis and metastatic progression in mice by amplifying ErbB2 signaling. J Clin Invest 118: 64-78, 2008.

10. Lu C, Shahzad MM, Wang H, et al: EphA2 overexpression promotes ovarian cancer growth. Cancer Biol Ther 7: 1098-1103, 2008.

11. Zelinski DP, Zantek ND, Stewart JC, Irizarry AR and Kinch MS: EphA2 overexpression causes tumorigenesis of mammary epithelial cells. Cancer Res 61: 2301-2306, 2001.

12. Zantek ND, Azimi M, Fedor-Chaiken M, Wang B, Brackenbury R and Kinch MS: E-cadherin regulates the function of the EphA2 receptor tyrosine kinase. Cell Growth Differ 10: 629-638, 1999.

13. Lin YG, Han LY, Kamat AA, et al: EphA2 overexpression is associated with angiogenesis in ovarian cancer. Cancer 109: 332-340, 2007. 
14. Han L, Dong Z, Qiao Y, et al: The clinical significance of EphA2 and Ephrin A-1 in epithelial ovarian carcinomas. Gynecol Oncol 99: 278-286, 2005

15. Brannan JM, Dong W, Prudkin L, et al: Expression of the receptor tyrosine kinase EphA2 is increased in smokers and predicts poor survival in non-small cell lung cancer. Clin Cancer Res 15: 4423-4430, 2009.

16. Liu F, Park PJ, Lai W, et al: A genome-wide screen reveals functional gene clusters in the cancer genome and identifies EphA2 as a mitogen in glioblastoma. Cancer Res 66: 10815-10823, 2006.

17. Taddei ML,Parri M, Angelucci A, et al: EphA2 induces metastatic growth regulating amoeboid motility and clonogenic potential in prostate carcinoma cells. Mol Cancer Res 9: 149-160, 2011.

18. Herrem CJ, Tatsumi T, Olson KS, et al: Expression of EphA2 is prognostic of disease-free interval and overall survival in surgically treated patients with renal cell carcinoma. Clin Cancer Res 11: 226-231, 2005.

19. Liu Y, Xie C, Zhang X, et al: Elevated expression of HMGB1 in squamous-cell carcinoma of the head and neck and its clinical significance. Eur J Cancer 46: 3007-3015, 2010.

20. Liu Y, Zhang X, Qiu Y, et al: Clinical significance of EphA2 expression in squamous-cell carcinoma of the head and neck. J Cancer Res Clin Oncol 137: 761-769, 2011.

21. Johnson FM, Garden AS, Palmer JL, et al: A phase I/II study of neoadjuvant chemotherapy followed by radiation with boost chemotherapy for advanced T-stage nasopharyngeal carcinoma. Int J Radiat Oncol Biol Phys 63: 717-724, 2005.

22. Liu Y, Yu C, Qiu Y, et al: Downregulation of EphA2 expression suppresses the growth and metastasis in squamous-cell carcinoma of the head and neck in vitro and in vivo. J Cancer Res Clin Oncol 138: 195-202, 2012.
23. Zhu G, Xiao D, Chen Q and Zhu Y: The expression and its potentially clinical significance of EphA2 in nasopharyngeal carcinoma. Lin Chung Er Bi Yan Hou Tou Jing Wai Ke Za Zhi 25: 827-829, 833, 2011 (In Chinese).

24. Abraham S, Knapp DW, Cheng L, et al: Expression of EphA2 and Ephrin A-1 in carcinoma of the urinary bladder. Clin Cancer Res 12: 353-360, 2006.

25. Brannan JM, Sen B, Saigal B, et al: EphA2 in the early pathogenesis and progression of non-small cell lung cancer. Cancer Prev Res (Phila) 2: 1039-1049, 2009.

26. Kinch MS, Moore MB and Harpole DH Jr: Predictive value of the EphA2 receptor tyrosine kinase in lung cancer recurrence and survival. Clin Cancer Res 9: 613-618, 2003.

27. King AD, Ahuja AT, Leung SF, et al: Neck node metastases from nasopharyngeal carcinoma: MR imaging of patterns of disease. Head Neck 22: 275-281, 2000.

28. Leung SF, Teo PM, Shiu WW, Tsao SY and Leung TW: Clinical features and management of distant metastases of nasopharyngeal carcinoma. J Otolaryngol 20: 27-29, 1991.

29. Ciuleanu TE, Fountzilas G, Ciuleanu E, Plataniotis M, Todor N and Ghilezan N: Paclitaxel and carboplatin in relapsed or metastatic nasopharyngeal carcinoma: a multicenter phase II study. J BUON 9: 161-165, 2004.

30. Landen CN Jr, Chavez-Reyes A, Bucana C, et al: Therapeutic EphA2 gene targeting in vivo using neutral liposomal small interfering RNA delivery. Cancer Res 65: 6910-6918, 2005. 